\title{
Ornithine decarboxylase and glutamate decarboxylase 65 as prognostic markers of gallbladder malignancy: A clinicopathological study in benign and malignant lesions of the gallbladder
}

\author{
XIAOFENG DENG and DONGNI PEI
}

Department of General Surgery, The Second Xiangya Hospital, Central South University, Changsha, Hunan 410011, P.R. China

Received July 3, 2012; Accepted October 31, 2012

DOI: $10.3892 / \mathrm{mmr} .2012 .1178$

\begin{abstract}
Ornithine decarboxylase (ODC) plays a critical role in cell proliferation and is overexpressed in a variety of cancers. Furthermore, $\gamma$-aminobutyric acid (GABA) content and glutamate decarboxylase (GAD) activity are increased in neoplastic tissues in colon and breast cancer. However, few studies have examined these molecules in gallbladder cancer specimens. We observed the expression levels of ODC and GAD65 in benign and malignant lesions of the gallbladder and investigated their clinicopathological significance for the first time. The expression levels of ODC and GAD65 in specimens from gallbladder adenocarcinoma $(n=108)$, peritumoral tissues $(n=46)$, adenomatous polyps $(n=15)$ and chronic cholecystitis $(n=35)$ were detected using immunohistochemical methods. Kaplan-Meier survival and Cox regression analyses were carried out to explore the clinical and pathological correlations. The levels of positive staining of ODC and GAD65 were significantly higher in gallbladder adenocarcinoma than in peritumoral tissues, adenomatous polyps and chronic cholecystitis. The Kaplan-Meier survival analysis and Cox regression analysis showed that the expression of ODC and GAD65 correlated significantly with the one-year survival rate and the mean survival time of the patients postoperatively. We conclude that the overexpression of ODC and GAD65 are significant in the carcinogenesis and progression of gallbladder adenocarcinoma. They may be important biological markers for the evaluation of biological behaviors and the prognosis of gallbladder adenocarcinoma.
\end{abstract}

\section{Introduction}

Gallbladder cancers are associated with a dismal prognosis, with a 5 -year survival rate of $<5 \%$ for those patients whose disease is

Correspondence to: Dr Dongni Pei, Department of General Surgery, The Second Xiangya Hospital, Central South University, 139 Renmin Road, Changsha, Hunan 410011, P.R. China

E-mail: 13787782059@163.com

Key words: ornithine decarboxylase, glutamate decarboxylase 65, gallbladder cancer, clinicopathology not amenable to surgery (1). The majority of gallbladder cancers are adenocarcinomas, with a small percentage being squamous cell carcinomas. Chronic cholecystitis and cholelithiasis are risk factors for primary carcinoma of the gallbladder (2). In certain parts of the world, the incidence is comparatively high and contributes to a social disaster as a result of its poor outcome.

The surgical management of gallbladder cancer has always been controversial. Surgeons wordwide have repeatedly stressed the need for complete surgical resection. However, even among patients who undergo radical surgical resection, the reported median survival across all stages is $35-38 \%$ (3). An improved understanding of the clinicopathological features of gallbladder carcinogenesis may provide useful insights in the development of methods for the diagnosis and treatment of this deadly disease.

Ornithine decarboxylase (ODC) is the first and rate-limiting enzyme in polyamine biosynthesis. Polyamines are known to play key roles in cell proliferation and other biological processes. Polyamine biosynthesis is closely associated with physiological cell growth, proliferation, regeneration and pathological proliferation. It is necessary for cells to progress into the $\mathrm{S}$ phase; polyamine depletion arrests cells in G1. Elevated levels of ODC have been identified in gastric cancer, glioma, breast cancer, colon cancer, pancreatic cancer, lung cancer and prostate cancer, and may contribute to their carcinogenesis (4-8). $\gamma$-aminobutyric acid (GABA) is synthesized by glutamate decarboxylase (GAD), which has two forms, GAD65 and GAD67. The former may be related to the carcinogenesis, progression and biological behaviors of a number of malignancies. Several previous studies have suggested a connection between the GABAergic system and neoplastic processes $(9,10)$. It has been confirmed that GABA content and GAD activity are increased in the neoplastic tissues in colon and breast cancer (11-13). In the current study, we observed the expression of ODC and GAD65 in gallbladder specimens by means of immunohistochemical analysis and analyzed the correlation between the expression levels of ODC and GAD65 and the clinicopathological features of the gallbladder lesions.

\section{Materials and methods}

Materials. A total of 108 gallbladder carcinoma specimens were collected from Xiangya Hospital, The Second Xiangya 
Hospital of Central South University and The People's Hospital of Hunan Province, Changsha, China. Of these, 77 specimens came from female patients and 31 from males. All specimens were diagnosed as adenocarcinomas, of which 9 had adenoma lesions, 29 were highly differentiated, 29 moderately differentiated, 30 poorly differentiated and the remaining 11 were mucous adenomas. Surgery revealed the invasion of pericholecystic tissues and organs in 59 cases, local lymph node metastases in 59 cases and gallstones/ cholelithiasis in 58 cases. Radical resection was applied to 34 cases and palliative resection/surgery to 48 cases while 26 cases were unresectable to surgery due to local invasion into critical structures or metastasis beyond regional confines. Data concerned with survival analysis were acquired for 67 cases by long-term follow-up of all patients, and the postoperative survival time was grouped as $\geq 1$ year (20 cases) and $<1$ year (47 cases). From the 108 gallbladder adenocarcinoma samples, we obtained 46 sections of peritumoral tissues (distance to adenocarcinomas $\geq 3 \mathrm{~mm}$ ), of which 10 were normal by pathological analysis and 10, 12 and 14 had mild, moderate and severe atypical proliferation, respectively. A total of 15 specimens of gallbladder diagnosed as adenomatous polyps were obtained from The Second Xiangya Hospital of Central South University, including specimens from 10 females and 5 males of average age 51.8 \pm 11.6 years (range 42-60 years). The adenomatous polyps were $8-15 \mathrm{~mm}$ in size, and 5 of the 15 had moderate to severe proliferation. In addition, 35 chronic cholecystitis specimens were obtained as controls, of which 15 were from patients with chronic cholecystitis alone and 20 were from patients with chronic cholecystitis and gallstones. Histologically, the 35 specimens included 11 with normal gallbladder mucosa, 12 with mild atypical proliferation, 7 with moderate atypical proliferation and 5 with severe atypical proliferation. All samples were fixed in $4 \%$ formaldehyde and $4-\mu \mathrm{m}$ sections were prepared for immunohistochemical studies. The study was approved by the ethics committee of Second Xiangya Hospital, Central South University, Changsha, China. Written informed patient consent was obtained from the patient's family.

Immunohistochemistry. For ODC and GAD65 detection, immunochemical staining was carried out using EnVision ${ }^{\mathrm{TM}}$ (ChemMate $^{\mathrm{TM}}$ EnVision +/HRP/DAB, rabbit/mouse two step staining method) according to the instructions of the manufacturer (Dako Laboratories, Inc., Santa Barbara, CA, USA). Briefly, paraffin-embedded gallbladder adenocarcinoma tissues were cut into $4-\mu \mathrm{m}$-thick sections. The sections were deparaffinized and incubated with $3 \% \mathrm{H}_{2} \mathrm{O}_{2}$ solution for $15 \mathrm{~min}$, followed by EDTA-trypsinase digestion $(0.125 \%$, $\mathrm{pH}$ 9.0) for $15 \mathrm{~min}$, then soaked with PBS (pH 7.4) 3 times, each for $5 \mathrm{~min}$. The pre-treated sections were then incubated with rabbit anti-human ODC or GDA65 (Dako Laboratories Inc.) for $60 \mathrm{~min}$ at room temperature. Solution A (ChemMate EnVision +/HRP) was added and incubation was continued for another $30 \mathrm{~min}$. Substrate DAB liquid was added and the sections were then hematoxylin counter-stained. The slides were dehydrated with different concentrations of alcohol and soaked in xylene for 5 min (3 times), and then mounted permanently with neutral balsam. The slides were examined independently by two pathologists. The results of ODC or
GAD65 immunochemical staining were considered to be positive when $>25 \%$ of the tumor cells were stained. The positive controls were provided by Bosite Inc. (Wuhan, China) and the negative controls used $0.01 \mathrm{~mol} / \mathrm{l} \mathrm{PBS}$ ( $\mathrm{pH}$ 7.4) solution (to substitute for the first antibody).

Statistical analysis. The SPSS 19.0 software was used for calculation of the correlations between the ODC or GAD65 levels and the histological or clinical factors by the $\chi^{2}$ independence test. Fisher's exact probability test was also used for analyzing statistical associations between two independent sample groups. $\mathrm{P}<0.05$ was considered to indicate a statistically significant result. Disease-specific overall survival analyses were determined and compared using the Kaplan-Meier method and the log-rank test. For multivariate analysis, the Cox regression method was employed. $95 \%$ confidence intervals (CIs) were used.

\section{Results}

Expression of ODC and GAD65 in human gallbladder adenocarcinoma, peritumoral tissues, adenomatous polyps and chronic cholecystitis. The number of cases staining positive for ODC and GAD65 were 65 and 59, respectively, among the 108 patients with gallbladder adenocarcinoma, 13 and 14, respectively, among the 46 peritumoral tissues, 3 and 4, respectively, among the 15 patients with adenomatous polyps and 4 and 6 , respectively, among the 35 patients with chronic cholecystitis (Figs. 1-4). The number of positive stainings of ODC and GAD65 in the gallbladder adenocarcinoma tissues were significantly higher than those in the peritumoral tissues $(\mathrm{P}<0.01)$, adenomatous polyps $(\mathrm{P}<0.01$ for $\mathrm{ODC}$; $\mathrm{P}<0.05$ for GAD65) and chronic cholecystitis $(\mathrm{P}<0.01)$. Moreover, mild, moderate or severe atypical proliferation was observed in all cases expressing ODC or GAD65.

Correlation of ODC and GAD65 expression with clinical and pathological features of gallbladder adenocarcinoma. The expression levels of ODC and GAD65 in cases with adenoma canceration or well-differentiated carcinoma or without lymph node metastasis or surrounding invasion were significantly lower than those in cases with poorly differentiated carcinoma, lymph node metastasis or surrounding invasion. No significant correlation was observed between the expression levels of ODC and GAD65 and the clinicopathological features of patients with gallbladder adenocarcinoma, including gender, age, tumor size and gallstones (Table I).

Correlation between disease-specific overall survival and expression of $O D C$ and GAD65. Following surgical resection, only 67 of the 108 patients were successfully followed up via telephone or mail surveys, among which 20 cases had survived $\geq 1$ year and 47 cases had succumbed to the disease $<1$ year postoperatively, with a mean survival time of $9.7 \pm 4.4$ months. Of the 67 surviving patients, 40 had positive immunohistochemical staining of ODC and 36 had positive staining of GAD65. The relevance of the patients' survival to the positive expression of ODC and GAD65 was examined by univariate Kaplan-Meier survival analysis. Overall survival was inversely associated with positive or increased expression of ODC 


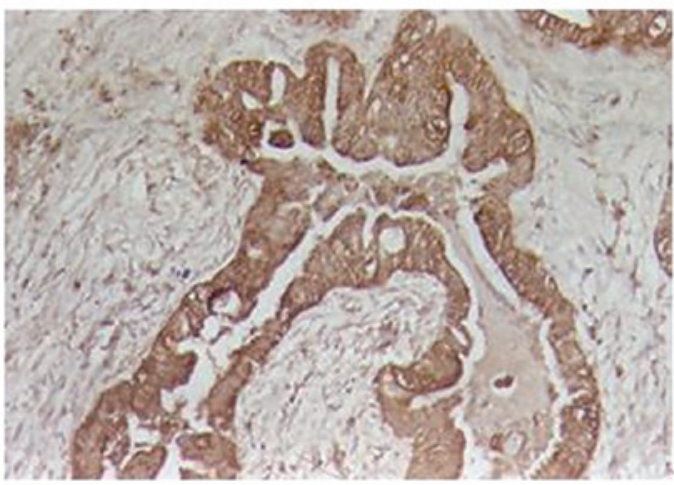

Figure 1. Positive expression of ornithine decarboxylase (ODC) in moderately differentiated adenocarcinoma. EnVison ${ }^{\mathrm{TM}}$ immunohistochemistry, $\mathrm{x} 200$.

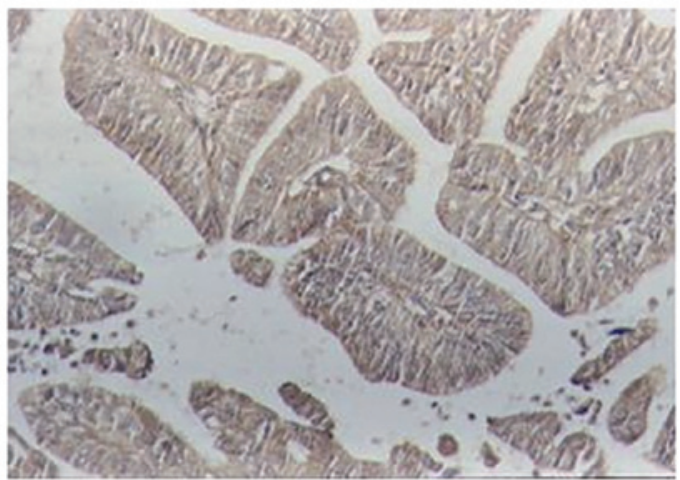

Figure 2. Positive expression of ornithine decarboxylase (ODC) in adenomatous polyp with severely atypical hyperplasia. EnVison ${ }^{\mathrm{TM}}$ immunohistochemistry, x200.

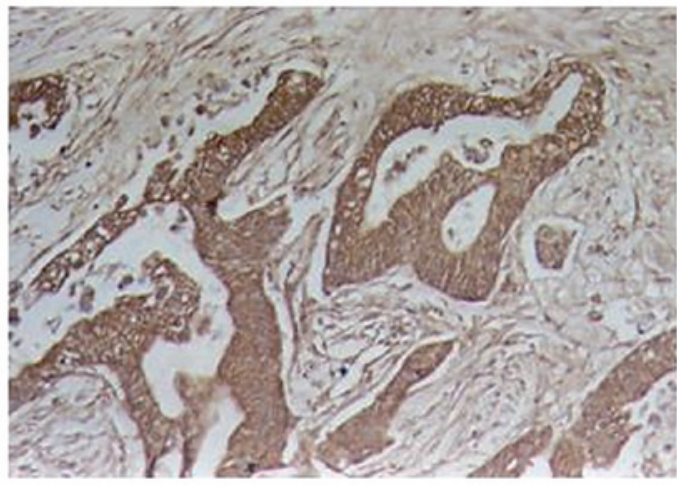

Figure 3. Positive expression of glutamate decarboxylase 65 (GAD65) in

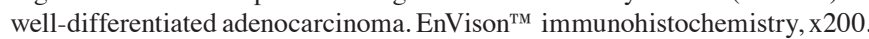

(Fig. 5; $\mathrm{P}=0.009$ ) and GAD65 (Fig. 6; $\mathrm{P}=0.006$ ). The relevance of overall survival to other clinicopathological characteristics was also assessed by univariate analysis, which revealed that the overall survival was associated with tumor pathological type $(\mathrm{P}<0.01)$, tumor diameter $(\mathrm{P}<0.01)$, lymph node metastasis $(\mathrm{P}<0.01)$ and surrounding tissue invasion $(\mathrm{P}<0.01)$. All factors that showed significant association in the univariate Kaplan-Meier analysis were subsequently subjected to multivariate Cox regression survival analysis, which indicated that tumor maximum diameter $\geq 2 \mathrm{~cm}$, lymph node metastasis and

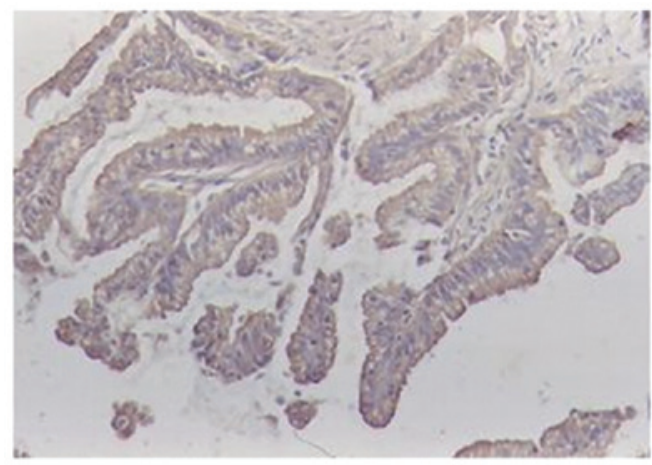

Figure 4. Positive expression of glutamate decarboxylase 65 (GAD65) in peritumoral tissue with moderately atypical hyperplasia. EnVison ${ }^{\mathrm{TM}}$ immunohistochemistry, $\mathrm{x} 200$.

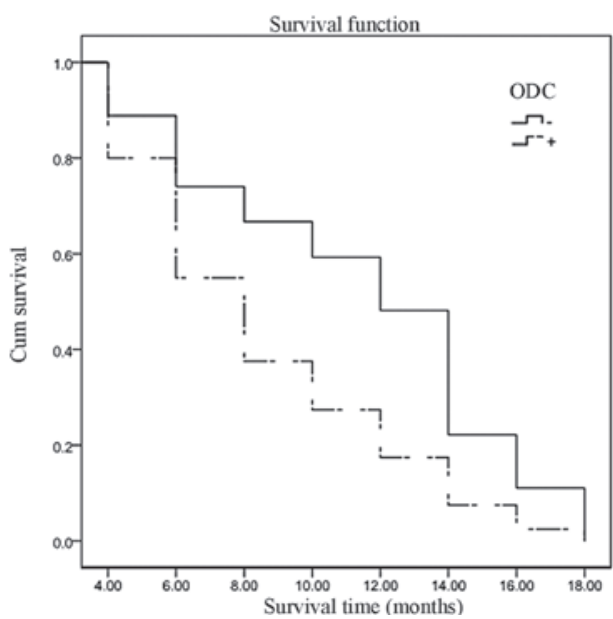

Figure 5. Univariate Kaplan-Meier survival analysis showed that overall survival was inversely associated with increased expression of ornithine decarboxylase (ODC), as illustrated by the dotted line $(\mathrm{P}=0.009)$.

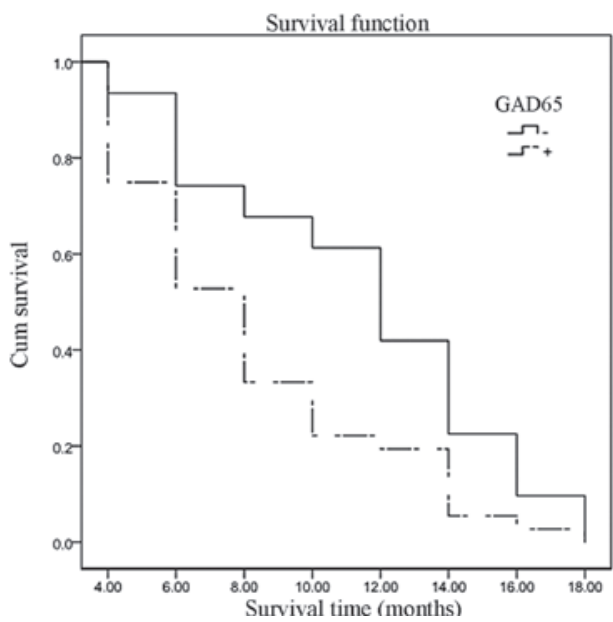

Figure 6. Univariate Kaplan-Meier survival analysis showed that overall survival was inversely associated with increased expression of glutamate decarboxylase 65 (GAD65) as illustrated by the dotted line $(\mathrm{P}=0.006)$.

surrounding tissue invasion followed by ODC or GAD65 positive expression were the most significant predictors of short overall survival (Table II). 
Table I. Correlation between gallbladder cancer and expression of ODC and GAD65.

\begin{tabular}{|c|c|c|c|c|c|}
\hline \multirow[b]{2}{*}{ Clinicopathological features } & \multirow[b]{2}{*}{ Total } & \multicolumn{2}{|c|}{ ODC } & \multicolumn{2}{|c|}{ GAD65 } \\
\hline & & Positive (\%) & P-value & Positive (\%) & P-value \\
\hline \multicolumn{6}{|l|}{ Gender } \\
\hline Male & 31 & $15(48.4)$ & \multirow[t]{2}{*}{$>0.05$} & $14(45.2)$ & \multirow[t]{2}{*}{$>0.05$} \\
\hline Female & 77 & $50(64.9)$ & & $45(58.4)$ & \\
\hline \multicolumn{6}{|l|}{ Age } \\
\hline$\leq 45$ & 24 & $15(62.5)$ & \multirow[t]{2}{*}{$>0.05$} & $12(50.0)$ & \multirow[t]{2}{*}{$>0.05$} \\
\hline$>45$ & 84 & $50(59.5)$ & & $47(56.0)$ & \\
\hline \multicolumn{6}{|l|}{ Pathological classification } \\
\hline Adenoma canceration & 9 & $3(33.3)$ & \multirow[t]{5}{*}{$<0.05$} & $2(22.2)$ & \multirow[t]{5}{*}{$<0.01$} \\
\hline Well-differentiated & 29 & $13(44.8)$ & & $11(37.9)$ & \\
\hline Moderately differentiated & 29 & $18(62.1)$ & & $16(55.2)$ & \\
\hline Poorly differentiated & 30 & $25(83.3)$ & & $24(80.0)$ & \\
\hline Mucous adenocarcinoma & 11 & $6(54.5)$ & & $6(54.5)$ & \\
\hline \multicolumn{6}{|l|}{ Tumor diameter } \\
\hline$<2 \mathrm{~cm}$ & 31 & $15(48.4)$ & \multirow[t]{2}{*}{$>0.05$} & $13(41.9)$ & \multirow[t]{2}{*}{$>0.05$} \\
\hline$\geq 2 \mathrm{~cm}$ & 77 & $50(64.9)$ & & $46(59.7)$ & \\
\hline \multicolumn{6}{|l|}{ Lymph node metastasis } \\
\hline No & 49 & $21(42.9)$ & \multirow[t]{2}{*}{$<0.01$} & $19(38.8)$ & \multirow[t]{2}{*}{$<0.01$} \\
\hline Yes & 59 & $44(74.6)$ & & $40(67.8)$ & \\
\hline \multicolumn{6}{|l|}{ Surrounding invasion } \\
\hline No & 49 & $22(44.9)$ & \multirow[t]{2}{*}{$<0.01$} & $20(40.8)$ & \multirow[t]{2}{*}{$<0.01$} \\
\hline Yes & 59 & $43(72.9)$ & & $39(66.1)$ & \\
\hline \multicolumn{6}{|l|}{ Gallstones } \\
\hline No & 50 & $26(52.0)$ & \multirow[t]{2}{*}{$>0.05$} & $23(46.0)$ & \multirow[t]{2}{*}{$>0.05$} \\
\hline Yes & 58 & $39(67.2)$ & & $36(62.1)$ & \\
\hline
\end{tabular}

ODC, ornithine decarboxylase; GAD65, glutamate decarboxylase 65.

Table II. Multivariate Cox regression analysis of overall survival in 67 patients with surgical resection of gallbladder carcinoma.

\begin{tabular}{|c|c|c|c|c|c|c|c|}
\hline \multirow[b]{2}{*}{ Factor } & \multirow[b]{2}{*}{ Category } & \multirow[b]{2}{*}{$\mathrm{B}$} & \multirow[b]{2}{*}{$\mathrm{SE}(\mathrm{B})$} & \multirow[b]{2}{*}{$\operatorname{Exp}(B)$} & \multirow[b]{2}{*}{$\mathrm{P}$} & \multicolumn{2}{|c|}{$95 \%$ CI for $\operatorname{Exp}(B)$} \\
\hline & & & & & & Inferior & Superior \\
\hline Pathology type & $\begin{array}{l}\text { Adenoma canceration/well-/moderately/ } \\
\text { poorly differentiated/mucous carcinoma }\end{array}$ & 0.208 & 0.123 & 1.231 & 0.091 & 0.967 & 1.567 \\
\hline Tumor diameter & $<2.0 \mathrm{~cm} / \geq 2.0 \mathrm{~cm}$ & 0.617 & 0.274 & 1.853 & 0.025 & 1.082 & 3.173 \\
\hline Lymph node metastasis & $\mathrm{No} / \mathrm{Yes}$ & 0.561 & 0.275 & 1.752 & 0.041 & 1.022 & 3.002 \\
\hline Surrounding tissue invasion & $\mathrm{No} / \mathrm{Yes}$ & 0.634 & 0.266 & 1.886 & 0.017 & 1.120 & 3.175 \\
\hline ODC & $-/+$ & 0.568 & 0.285 & 1.765 & 0.046 & 1.011 & 3.084 \\
\hline GAD65 & $-/+$ & 0.585 & 0.269 & 1.795 & 0.030 & 1.059 & 3.042 \\
\hline
\end{tabular}

ODC, ornithine decarboxylase; GAD65, glutamate decarboxylase 65; CI, confidence interval.

\section{Discussion}

ODC, a key enzyme in polyamine metabolism, is observed in numerous types of cells from humans and animals. It is a dimer consisting of two 50-kDa subunits. The ODC-related sequences, including ODC1 and ODC2, are present on human chromosomes 2 p25 and 7 q31 respectively. ODC1 is an important functional gene which exists universally in organisms, whereas ODC2 is a pseudogene which exists only in the human body and is without a clear function. The 
degradation of ODC depends mainly on ODC antizyme (OAZ) which functions not by suppressing the synthesis of ODC but by formulation of an ODC-antizyme complex. The latter is generated to promote the degradation of ODC by the $26 \mathrm{~S}$ proteasome and polyamine synthesis is subsequently decreased. Polyamines have been shown to be closely associated with cell growth, proliferation, division, differentiation and malignant transformation. As the first rate-limiting enzyme in polyamine synthesis, ODC has a significant effect on polyamine levels. ODC is overexpressed in cancerous tissues from a number of malignancies, including gastric cancer, pancreatic cancer and colorectal cancer $(5,6,14)$, and the inhibition of ODC may be a therapeutic strategy for malignancies, including breast cancer (15). It has been reported that the overexpression of ODC may lead to increased polyamine levels and thus induce the overproliferation and malignant transformation of cells (4,16-19). It contributes to the development, progression, metastasis and surrounding invasion of malignant tumors, and serves as a useful biological marker for certain precancerous lesions of digestive malignancies $(4-8,16,17,20)$. A previous study demonstrated that Helicobacter pylori upregulates the expression of ODC, resulting in gastric precursor lesions and cancer (21). Carcinomas with highly expressed ODC are more progressive and poorly differentiated, with increased metastasis, surrounding invasion and poor prognosis. Hence, ODC may be a novel target for use in the development of new anticancer drugs and as an indicator for the efficacy of treatments $(4-8,16,17,20)$.

Tissue-specific markers are useful for the identification of tumor type in advanced cancers of unknown origin. Jaraj et al investigated the expression of GAD67 (GAD1) in the prostate and found that GAD67 expression was significantly higher in malignant and benign prostatic tissue than in non-prostatic control tissues. It was suggested that GAD67 may serve as a highly prostate-specific tissue biomarker (22). However, whether GAD 65 (GAD2) plays a similar role in malignancies of the gallbladder remains unknown. In our study, we identified that GAD65 expression levels were significantly higher in gallbladder malignancy than in benign tissue.

Previous studies have shown that GABA stimulates the growth of a variety of types of cancer $(23,24)$. The key enzyme for GABA synthesis is GAD which appears in two isoforms, GAD65 and GAD67, named according to their molecular weights (25). According to a study by Moon et al, the proliferation of HT-29 colon cancer cells was significantly inhibited when GAD67 expression was repressed (26). GAD65 is overexpressed in the cancerous tissues of numerous malignancies, including colon cancer, breast cancer and gastric cancer, and is closely associated with the clinicopathology of the diseases. Increased levels of GAD65 expression represent a more malignant, progressive and invasive nature of the lesion $(11-13,27)$. The administration of a GABA receptor antagonist inhibits the growth and development of numerous types of cells, including malignant cells $(9,10)$.

In the present study, the positive staining of ODC and GAD65 observed in gallbladder adenocarcinoma was significantly higher than in peritumoral tissues, adenomatous polyps and chronic cholecystitis. Furthermore, each case with expression of ODC and GAD65 was accompanied by mild, moderate or severe atypical proliferation of the gallbladder mucosal epithelium. As revealed in the present study, the expression levels of ODC and GAD65 in cases with adenoma canceration or well-differentiated carcinoma or without lymph node metastasis or surrounding invasion were significantly lower than those in cases with poorly differentiated carcinoma, lymph node metastasis or surrounding invasion. The expression levels of ODC and GAD65 in cases surviving >one year were significantly lower than those in cases succumbing to the disease within one year. Cox regression analysis revealed that the expression levels of ODC and GAD65 were ideal prognostic markers in gallbladder carcinoma. These results are in accordance with findings reported from previous studies. This suggests that ODC and GAD65 are closely correlated with the development, progression, metastasis and surrounding invasion of gallbladder adenocarcinoma and may be important biological markers for the early screening of gallbladder carcinoma in benign lesions and for the prognosis of gallbladder malignancy.

\section{Acknowledgements}

The authors would like to thank Professor Zhulin Yang for designing the study and examining the specimens.

\section{References}

1. Ito H, Matros E, Brooks DC, Osteen RT, Zinner MJ, Swanson RS, Ashley SW and Whang EE: Treatment outcomes associated with surgery for gallbladder cancer: a 20 -year experience. J Gastrointest Surg 8: 183-190, 2004.

2. Randi G, Franceschi S and La Vecchia C: Gallbladder cancer worldwide: geographical distribution and risk factors. Int J Cancer 118: 1591-1602, 2006.

3. Shukla PJ and Barreto SG: Gallbladder cancer: we need to do better! Ann Surg Oncol 16: 2084-2085, 2009.

4. Gerner EW and Meyskens FL Jr: Polyamines and cancer: old molecules, new understanding. Nat Rev Cancer 4: 781-792, 2004.

5. Miao XP, Li JS, Li HY, Zeng SP, Zhao Y and Zeng JZ: Expression of ornithine decarboxylase in precancerous and cancerous gastric lesions. World J Gastroenterol 13: 2867-2871, 2007.

6. Subhi AL, Tang B, Balsara BR, et al: Loss of methylthioadenosine phosphorylase and elevated ornithine decarboxylase is common in pancreatic cancer. Clin Cancer Res 10: 7290-7296, 2004.

7. Love RR, Astrow SH, Cheeks AM and Havighurst TC: Ornithine decarboxylase (ODC) as a prognostic factor in operable breast cancer. Breast Cancer Res Treat 79: 329-334, 2003.

8. Young L, Salomon R, Au W, Allan C, Russell P and Dong Q: Ornithine decarboxylase (ODC) expression pattern in human prostate tissues and ODC transgenic mice. J Histochem Cytochem 54: 223-229, 2006.

9. Matsuba T, Yano M, Abiru N, Takino H, Akazawa S, Nagataki S and Yasukawa K: Expression of recombinant human glutamic acid decarboxylase (GAD) in myeloma cells and enzyme-linked immunosorbent assay (ELISA) for autoantibodies to GAD. J Biochem 121: 20-24, 1997.

10. Pinal CS, Cortessis V and Tobin AJ: Multiple elements regulate GAD65 transcription. Dev Neurosci 19: 465-475, 1997.

11. Matuszek M, Jesipowicz M and Kleinrok Z: GABA content and GAD activity in gastric cancer. Med Sci Monit 7: 377-381, 2001.

12. Opolski A, Mazurkiewicz M, Wietrzyk J, Kleinrok Z and Radzikowski C: The role of GABA-ergic system in human mammary gland pathology and in growth of transplantable murine mammary cancer. J Exp Clin Cancer Res 19: 383-390, 2000.

13. Kleinrok Z, Matuszek M, Jesipowicz J, Matuszek B, Opolski A and Radzikowski C: GABA content and GAD activity in colon tumors taken from patients with colon cancer or from xenografted human colon cancer cells growing as s.c. tumors in athymic nu/ nu mice. J Physiol Pharmacol 49: 303-310, 1998. 
14. Kumar KN, Raja SB, Vidhya N and Devaraj SN: Ellagic acid modulates antioxidant status, ornithine decarboxylase expression, and aberrant crypt foci progression in 1,2-dimethylhydrazineinstigated colon preneoplastic lesions in rats. J Agric Food Chem 60: 3665-3672, 2012.

15. Arisan ED, Obakan P, Coker A and Palavan-Unsal N: Inhibition of ornithine decarboxylase alters the roscovitine-induced mitochondrial-mediated apoptosis in MCF-7 breast cancer cells. Mol Med Rep 5: 1323-1329, 2012.

16. Manni A, Washington S, Griffith JW, et al: Influence of polyamines on in vitro and in vivo features of aggressive and metastatic behavior by human breast cancer cells. Clin Exp Metastasis 19: 95-105, 2002.

17. Kubo S, Tamori A, Tanaka H, et al: Polyamine metabolism and recurrence after resection for hepatocellular carcinoma. Hepatogastroenterology 51: 208-210, 2004.

18. Smirnova OA, Isaguliants MG, Hyvonen MT, et al: Chemically induced oxidative stress increases polyamine levels by activating the transcription of ornithine decarboxylase and spermidine/ spermine-N(1)-acetyltransferase in human hepatoma HUH7 cells. Biochimie 94: 1876-1883, 2012.

19. Barry EL, Mott LA, Sandler RS, Ahnen DJ and Baron JA: Variants downstream of the ornithine decarboxylase gene influence risk of colorectal adenoma and aspirin chemoprevention. Cancer Prev Res (Philadelphia) 4: 2072-2082, 2011.

20. Wolf C, Brüss M, Hänisch B, Göthert M, von Kügelgen I and Molderings GJ: Molecular basis for the antiproliferative effect of agmatine in tumor cells of colonic, hepatic, and neuronal origin Mol Pharmacol 71: 276-283, 2007.
21. Xu X, Liu Z, Fang M, et al: Helicobacter pylori CagA induces ornithine decarboxylase upregulation via Src/MEK/ERK/c-Myc pathway: implication for progression of gastric diseases. Exp Biol Med (Maywood) 237: 435-441, 2012.

22. Jaraj SJ, Augsten M, Häggarth L, Wester K, Pontén F, Ostman A and Egevad L: GAD1 is a biomarker for benign and malignant prostatic tissue. Scand J Urol Nephrol 45: 39-45, 2011.

23. Takehara A, Hosokawa M, Eguchi $\mathrm{H}$, Ohigashi $\mathrm{H}$, Ishikawa $\mathrm{O}$, Nakamura $\mathrm{Y}$ and Nakagawa H: Gamma-aminobutyric acid (GABA) stimulates pancreatic cancer growth through overexpressing GABAA receptor pi subunit. Cancer Res 67: 9704-9712, 2007.

24. Maemura K, Shiraishi N, Sakagami K, et al: Proliferative effects of gamma-aminobutyric acid on the gastric cancer cell line are associated with extracellular signal-regulated kinase $1 / 2$ activation. J Gastroenterol Hepatol 24: 688-696, 2009.

25. Walls AB, Nilsen LH, Eyjolfsson EM, et al: GAD65 is essential for synthesis of GABA destined for tonic inhibition regulating epileptiform activity. J Neurochem 115: 1398-1408, 2010.

26. Moon MS, Cho EW, Byun HS, Jung IL and Kim IG: GAD 67KD antisense in colon cancer cells inhibits cell growth and sensitizes to butyrate and $\mathrm{pH}$ reduction and $\mathrm{H}_{2} \mathrm{O}_{2}$ and gamma-radiation. Arch Biochem Biophys 430: 229-236, 2004.

27. Mazurkiewicz M, Opolski A, Wietrzyk J, Radzikowski C and Kleinrok Z: GABA level and GAD activity in human and mouse normal and neoplastic mammary gland. J Exp Clin Cancer Res 18: $247-253,1999$. 